\title{
Successful Treatment of Recalcitrant Prurigo with Alitretinoin
}

\author{
Karolina Gadaldi ${ }^{\mathrm{a}} \quad$ Yeliz Erdemoğlu $^{\mathrm{b}}$ Nikhil Yawalkar ${ }^{\mathrm{a}}$ \\ a Department of Dermatology, Inselspital, Bern University Hospital, University of Bern, Bern, Switzerland; \\ ${ }^{b}$ Department of Dermatology, School of Medicine, Acibadem University, Istanbul, Turkey
}

\section{Key Words}

Alitretinoin · Pruritus · Prurigo · Psoriasis

\begin{abstract}
Background: Chronic itch with secondary scratch lesions such as prurigo has a major impact on quality of life. Due to its relapsing nature and often unknown origin, its treatment is challenging. Objective: We sought to demonstrate that alitretinoin can be an efficacious and well-tolerated treatment in a patient suffering from chronic itch with concomitant prurigo and psoriatic lesions. Methods: Case report. Results: After 1 month of alitretinoin treatment ( $30 \mathrm{mg}$ daily), itch as well as prurigo and psoriasis lesions decreased markedly. Three cycles of alitretinoin were administered, as each cessation of treatment led to relapse of the symptoms after $6-8$ weeks. Tapering of the alitretinoin dose (30 mg every second day) after the third cycle allowed to maintain the effects for over 18 months. Conclusion: Treatment of refractory prurigo with alitretinoin might be an efficacious alternative to standard therapies. In case of relapse, retreatment with alitretinoin reinduces a further long-lasting response.
\end{abstract}

(c) 2015 S. Karger AG, Basel

\section{Introduction}

Chronic pruritus presenting with secondary scratch lesions such as prurigo has been demonstrated to have a major impact on quality of life. Although the underlying origin is often unknown, it may be associated with numerous dermatological, systemic and/or psychological diseases. Given its chronicity and relapsing nature, treatment of chronic pruritus with prurigo can be challenging. Temporary relief can be achieved by use of corticosteroids, topical calcineurin inhibitors, menthol, capsaicin creams or ultraviolet therapy. Treatment with oral agents such as ciclosporin, anxiolytics, opiate receptor antagonists, thalidomide and gabapentin can be satisfactory; however, their pronounced toxicity prevents the long-term use of these drugs, and the severity of the disease often worsens markedly after treatment has been stopped. We present a case of a patient suffering from chronic itch with concomitant prurigo and psoriasis lesions successfully treated with alitretinoin.

\section{Case Report}

A 46-year-old Caucasian woman, with a history of plaque psoriasis of the elbows and knees, chronic alcohol abuse and depression was admitted to our outpatient clinic with severe, 5-year pruritus and secondary prurigo lesions on the extensor surface of her arms and legs (fig. 1a, b). Physical examination of the patient, blood chemistry test, chest X-ray, as well as direct and indirect immunofluorescence did not confirm any underlying systemic disease. His- tological examination of a skin biopsy of a nodular lesion was consistent with prurigo.

Previous treatments including topical corticosteroids, topical tacrolimus, menthol, phototherapy, antihistamines, montelukast, doxepin and gabapentin did not provide healing of the lesions. Treatment with ciclosporin (200 mg daily) led to significant improvement of both prurigo and psoriatic lesions (body surface area: 3\%), but due to a potential risk of long-term adverse reactions the treatment was interrupted after 1 year.

Eventually, successful treatment with alitretinoin (30 mg daily) was introduced. After approximately 1 month, pruritus decreased and both prurigo and psoriasis lesions (body surface area: $<1 \%$ ) improved significantly (fig. 1c, d). After 5 months, the skin lesions healed completely with some postinflammatory pigmentations and scars (fig. 1e, f). Consequently, alitretinoin was stopped. Altogether 3 cycles of alitretinoin were administered, as each cessation of retinoid treatment led to relapse of the symptoms after approximately 6-8 weeks. Tapering of the alitretinoin dose (30 mg every second day) after the third cycle allowed to maintain the therapeutic effect for over 18 months. In the last 3 months treatment with alitretinoin $30 \mathrm{mg}$ was extended to every third day and no relapse has been observed so far. Treatment was well tolerated except for a slightly elevated level of nonfasting total cholesterol values (up to $7 \mathrm{mmol} / \mathrm{l}$, normal value: $<5.2 \mathrm{mmol} / \mathrm{l}$ ).

\section{KARGER 125}

(c) 2015 S. Karger AG, Base

1018-8665/15/2314-0330\$39.50/0
Karolina Gadaldi

Department of Dermatology, Inselspital

Bern University Hospital, University of Bern

$\mathrm{CH}-3010$ Bern (Switzerland)

E-Mailkarolina.gadaldi@insel.ch 

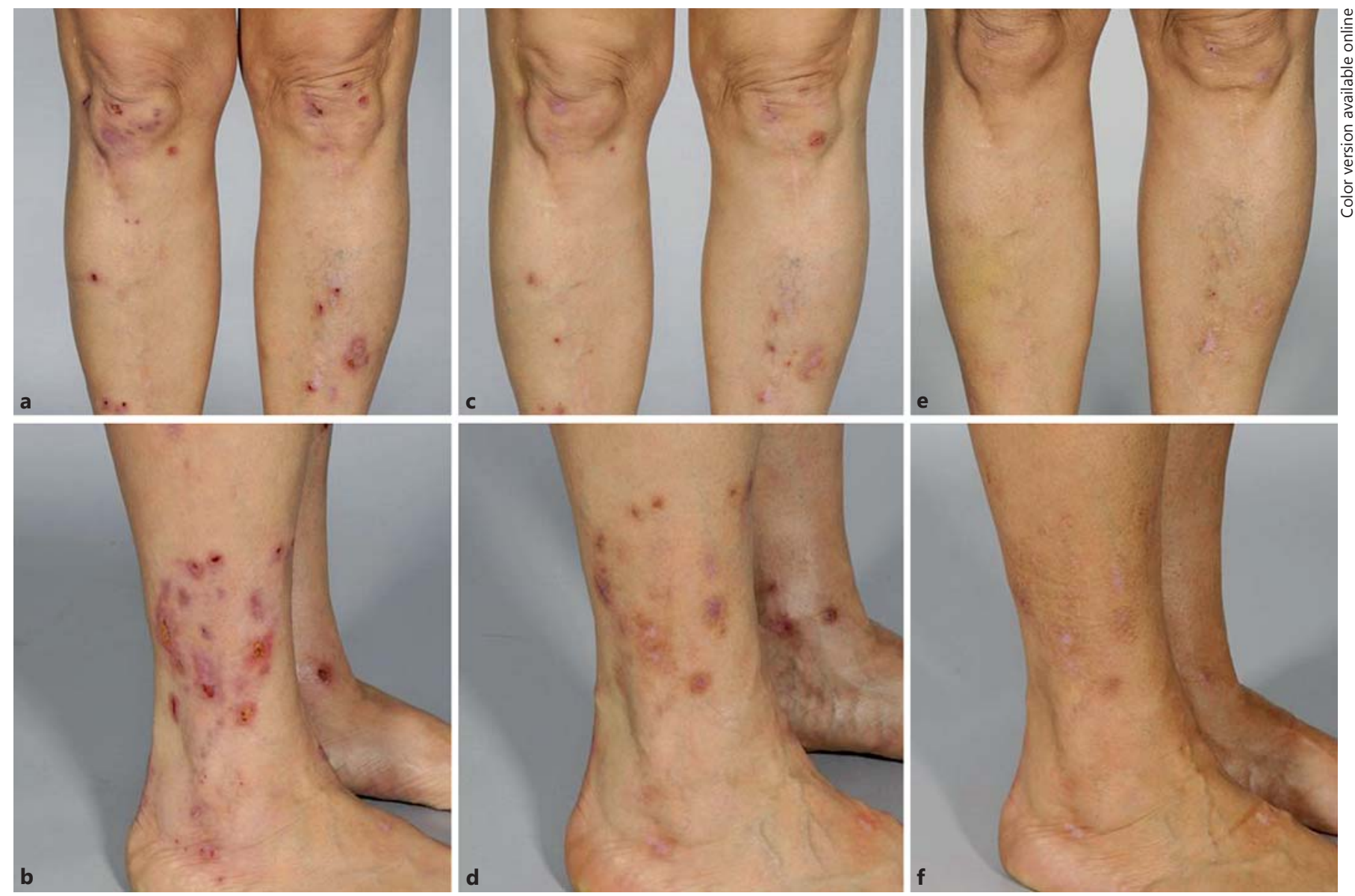

Fig. 1. Clinical presentation before (a, b), and outcome after approximately 1 month $(\mathbf{c}$, d) and after 5 months $(\mathbf{e}, \mathbf{f})$ of alitretinoin treatment.

\section{Discussion}

Alitretinoin (9-cis-retinoic acid) is a novel vitamin A derivate that binds to all 6 retinoid receptors (retinoic acid receptors RAR- $\alpha,-\beta,-\gamma$ and retinoid $\mathrm{X}$ receptors RXR- $\alpha,-\beta,-\gamma$ ). The most frequent retinoid receptor in the skin is RAR- $\gamma /$ RXR- $\alpha$ heterodimer [1]. Previously used retinoids (such as acitretin and etretinate) target mainly RAR receptors. Hence, alitretinoin is expected to act in a wider spectrum of diseases and on different pathways compared to the old retinoids. It has anti-inflammatory and immunomodulating effects and has been demonstrated to regulate the production of cytokine and leukocyte activity [2].

The most frequent adverse effects of alitretinoin include mucocutaneous dryness and headache [3]. The latter is dose dependent and often disappears with dose reduc- tion. Laboratory abnormalities comprise the typical effects of retinoids such as increase in serum lipids and liver enzymes, as well as a reduced thyroid-stimulating hormone level. In our case, except for a slightly elevated level of nonfasting total cholesterol there was no change in the laboratory investigations, especially no increase in liver values was observed. Furthermore, the mood of the patient was not altered during the therapy.

Alitretinoin has been registered as a systemic treatment for severe chronic hand eczema unresponsive to potent topical corticosteroids $[4,5]$. In addition to its approved use, alitretinoin has been reported to be beneficial also in refractory pityriasis rubra pilaris [6], lichen planus [7, 8], cutaneous lupus erythematosus [9], palmoplantar pustular psoriasis [10] as well as in mycosis fungoides [11], but it appeared to have a mixed effect in congenital ichthyosis
[12]. Several studies have also been carried out with alitretinoin used in the treatment of AIDS-related Kaposi's sarcoma [13].

The present report describes a case of chronic itch with secondary prurigo lesions and psoriasis successfully treated with alitretinoin (30 mg daily). After approximately 1 month of the therapy, pruritus decreased in our patient and both prurigo and psoriasis lesions improved. A significant decrease in intensity of itch after treatment with alitretinoin has already been demonstrated in the literature. A case report on 2 patients with mycosis fungoides described a cessation of pruritus after 2 months of alitretinoin treatment (30 mg daily), before improvement of the skin lesions [11]. Similarly, in 1 patient with lichen planus treated with alitretinoin (30 mg daily) pruritus disappeared after 4 weeks, before all skin lesions completely healed [8]. It is known that itch and subsequent repeated scratch- 
ing are major factors responsible for the development of prurigo lesions. Reduction of itching is therefore one of the main therapeutic strategies in this skin pathology.

Histological examinations of prurigo nodules have demonstrated a characteristic cellular pattern found in the majority of specimens. It consisted of fibrosis of the papillary dermis, an increased number of fibroblasts and capillaries, and a superficial, perivascular or interstitial inflammatory infiltrate of lymphocytes, macrophages and also eosinophils and neutrophils [14]. Moreover, it has been shown that an accumulation of neuropeptides upregulated the production of many proinflammatory cytokines [interleukin (IL)- $1 \alpha$, IL-1 $\beta$ and IL-8) as well as degranulation of an increased number of mast cells with subsequent release of histamine. The latter, in addition to induction of itch, stimulates proliferation of fibroblasts and synthesis of collagen.

The exact effects of alitretinoin on the pathomechanisms of itch and prurigo have not been elucidated so far. Apart from its well-known immunomodulatory effects on keratinocytes [15], it may also influence fibroblasts [16] and mast cells. Studies show that 9-cis-retinoic acid is able to inhibit in vitro the production of human mast cells [17]. Furthermore, in vitro stimulation of human $\mathrm{T}$ cells with 9-cis-retinoic acid increases the production of IL-4 [18]. The latter has been demonstrated to exert a potent inhibitory action on growth of mast cells [19]. It has previously been suggested that also pruritogenic IL-31 may play a role in the pathogenesis of itch in prurigo [20]. Among the sources of this cytokine are mast cells [21]. Based on the studies mentioned above, we can hypothesize that alitretinoin can reduce the production of IL31 through its inhibitory effect on mast cells.

Apart from an increased secretion of IL-4, in vitro stimulation of human T cells with 9-cis-retinoic acid resulted also in raised levels of IL-5 and IL-13, and decreased levels of interferon- $\gamma$, IL-2, IL12 p70 and tumor necrosis factor- $\alpha$ [18]. The latter and IL-12/IL-23 play an important role in the development of psoriasis $[22,23]$. Hence, it can explain the positive therapeutic effect of alitretinoin not only on prurigo, but also on psoriatic lesions in our patient. The positive effect of alitretinoin in psoriasis has been described previously in only few reports. Irla et al. [10] observed 50\% improvement in the Palmoplantar Psoriasis Area and Severity Index score in $100 \%$ of patients with palmoplantar pustular psoriasis $(n=7)$ treated with alitretinoin $30 \mathrm{mg}$ daily for 3 months. Moreover, they have also observed a significant decrease in itch intensity in all studied psoriatic patients.

After interruption of the therapy with alitretinoin, prurigo lesions relapsed in our patient within $6-8$ weeks. This phenome- non has already been observed in studies on hand eczema. The median time to relapse, defined as recurrence of $75 \%$ of initial signs and symptoms, was 5.5-6.2 months in the absence of other medications [4]. In our case the second and third therapeutic course of alitretinoin $(30 \mathrm{mg}$ daily) was necessary to completely heal the skin lesions. This is consistent with the data from a recently reported trial on chronic hand eczema [3]. The authors of this study demonstrated that alitretinoin at the dose of $30 \mathrm{mg}$ daily reinduced response in the majority of patients who had relapsed within 6 months following successful treatment with an initial course of alitretinoin.

In conclusion, our report shows that treatment of refractory prurigo with alitretinoin might be an efficacious alternative to standard topical and systemic treatments. In case of relapse, retreatment with alitretinoin reinduces a further long-lasting response. Further clinical studies are warranted to confirm efficacy and safety of alitretinoin in this disease.

\section{Disclosure Statement}

N. Yawalkar has served as a consultant and/or has received research trial support from Basilea and GSK. K. Gadaldi and Y. Erdemoğlu have no financial interest or affiliations to disclose.

\section{References}

1 Cheng C, Michaels J, Scheinfeld N: Alitretinoin: a comprehensive review. Expert Opin Investig Drugs 2008;17:437-443.

-2 Cheer SM, Foster RH: Alitretinoin. Am J Clin Dermatol 2000;1:307-314.

-3 Bissonnette R, Worm M, Gerlach B, et al: Successful retreatment with alitretinoin in patients with relapsed chronic hand eczema. $\mathrm{Br}$ J Dermatol 2010;162:420-426.

4 Ruzicka T, Lynde CW, Jemec GB, et al: Efficacy and safety of oral alitretinoin (9-cis retinoic acid) in patients with severe chronic hand eczema refractory to topical corticosteroids: results of a randomized, double-blind, placebo-controlled, multicentre trial. $\mathrm{Br} \mathrm{J}$ Dermatol 2008;158:808-817.

5 Dirschka T, Reich K, Bissonnette R, et al: An open-label study assessing the safety and efficacy of alitretinoin in patients with severe chronic hand eczema unresponsive to topical corticosteroids. Clin Exp Dermatol 2011;36: 149-154.
-6 Schmitt L, Inhoff O, Dippel E: Oral alitretinoin for the treatment of recalcitrant pityriasis rubra pilaris. Case Rep Dermatol 2011;3:8588.

7 Kolios AG, Marques Maggio E, Gubler C, et al: Oral, esophageal and cutaneous lichen ruber planus controlled with alitretinoin: case report and review of the literature. Dermatology 2013;226:302-310.

8 Brehmer F, Haenssle HA, Schön MP, et al: Response of recalcitrant lichen planus to alitretinoin in 3 patients. J Am Acad Dermatol 2011;65:58-60.

-9 Kuhn A, Patsinakidis N, Luger T: Alitretinoin for cutaneous lupus erythematosus. J Am Acad Dermatol 2012;67:123-126.

10 Irla N, Navarini AA, Yawalkar N: Alitretinoin abrogates innate inflammation in palmoplantar pustular psoriasis. Br J Dermatol 2012;167: 1170-1174.
11 Coors EA, von den Driesch P: Treatment of 2 patients with mycosis fungoides with alitretinoin. J Am Acad Dermatol 2012;67:265-267.

12 Gånemo A, Sommerlund M, Vahlquist A: Oral alitretinoin in congenital ichthyosis: a pilot study shows variable effects and a risk of central hypothyroidism. Acta Derm Venereol 2012;92:256-257.

13 Bodsworth NJ, Bloch M, Bower M, et al: Phase III vehicle-controlled, multi-centered study of topical alitretinoin gel $0.1 \%$ in cutaneous AIDS-related Kaposi's sarcoma. Am J Clin Dermatol 2001;2:77-87.

14 Weigelt N, Metze D, Ständer S: Prurigo nodularis: systematic analysis of 58 histological criteria in 136 patients. J Cutan Pathol 2010;37: 578-586.

15 Gibbs S, Backendorf C, Ponec M: Regulation of keratinocyte proliferation and differentiation by all-trans-retinoic acid, 9-cis-retinoic acid and 1,25-dihydroxy vitamin $\mathrm{D}_{3}$. Arch Dermatol Res 1996;288:729-738. 
16 Xiao R, Kanekura T, Yoshida N, et al: 9-cisretinoic acid exhibits antifibrotic activity via the induction of cyclooxygenase-2 expression and prostaglandin $\mathrm{E}_{2}$ production in scleroderma fibroblasts. Clin Exp Dermatol 2008; 33:484-490.

$\checkmark 17$ Kinoshita T, Koike K, Mwamtemi HH, et al: Retinoic acid is a negative regulator for the differentiation of cord blood-derived human mast cell progenitors. Blood 2000;95:28212828.

18 Dawson HD, Collins G, Pyle R, et al: Direct and indirect effects of retinoic acid on human Th2 cytokine and chemokine expression by human T lymphocytes. BMC Immunol 2006; $7: 27$.

19 Sillaber C, Sperr WR, Agis H, et al: Inhibition of stem cell factor dependent formation of human mast cells by interleukin- 3 and interleukin-4. Int Arch Allergy Immunol 1994;105: 264-268.

20 Sonkoly E, Muller A, Lauerma AI, et al: IL-31: a new link between $\mathrm{T}$ cells and pruritus in atopic skin inflammation. J Allergy Clin Immunol 2006;117:411-417.

21 Niyonsaba F, Ushio H, Hara M, et al: Antimicrobial peptides human beta-defensins and cathelicidin LL-37 induce the secretion of a pruritogenic cytokine IL-31 by human mast cells. J Immunol 2010;184:3526-3534.

22 Nair RP, Duffin KC, Helms C, et al: Genomewide scan reveals association of psoriasis with IL-23 and NF-kappaB pathways. Nat Genet 2009;41:199-204.

23 Tausend W, Downing C, Tyring S: Systematic review of interleukin-12, interleukin-17, and interleukin-23 pathway inhibitors for the treatment of moderate-to-severe chronic plaque psoriasis: ustekinumab, briakinumab, tildrakizumab, guselkumab, secukinumab, ixekizumab, and brodalumab. J Cutan Med Surg 2014; 18:156-169. 